\title{
Gravitons into Gravitational Field
}

\author{
Andrey N. Volobuev \\ Department of Medical Physics, Samara State Medical University, Samara, Russia \\ Email: volobuev47@yandex.ru
}

How to cite this paper: Volobuev, A.N. (2018) Gravitons into Gravitational Field. Journal of High Energy Physics, Gravitation and Cosmology, 4, 695-715.

https://doi.org/10.4236/jhepgc.2018.44039

Received: August 31, 2018

Accepted: October 8, 2018

Published: October 11, 2018

Copyright $\odot 2018$ by author and Scientific Research Publishing Inc. This work is licensed under the Creative Commons Attribution International License (CC BY 4.0).

http://creativecommons.org/licenses/by/4.0/

\begin{abstract}
The problems connected to propagation of a gravitational field are considered. The constant homogeneous gravitational field is investigated. The law of electromagnetic radiation frequency change in this gravitational field is shown. On the basis of the solution of the Einstein's equation for a weak gravitational field, the flux of gravitational radiation energy from system of cooperating masses is found. The equation for gravitational waves is found. On the basis of refusal from a stresses tensor into energy-impulse tensor and use of a quantum gravitational eikonal, the quantum form of the energy-impulse tensor in Einstein's equation is found. The equation for a graviton propagating in a gravitational field of a double star is found. Resonant interaction of a graviton and a gravitational field of a double star are investigated. It is shown that such interaction allows registering the gravitons.
\end{abstract}

\section{Keywords}

A Gravitational Eikonal, Metric Tensor, Einstein's Equation, Energy Flux, Gravitational Waves, Energy-Impulse Tensor, Registration of Gravitons

\section{Introduction}

The modern theory of gravitation - the theory general relativity of Einstein -is a basis for calculation of the astrophysics phenomena. It is generalization Newtonian dynamics, including the law of universal gravitation. As well as Newtonian dynamics the theory general relativity is not the quantum theory. The Einstein's equation for a gravitational field does not have stochastic nature. It contradicts modern physics. For example, for an electron cooperating with a gravitational field with help of the Einstein's equation, it is possible to calculate position absolutely precisely that contradicts a principle of Heisenberg's uncertainty.

Obviously such situation is unacceptable however the problem of a gravitational field quantization till now is not solved though for the solving of this 
problem many efforts have been applied [1] [2] [3] [4].

Recently the problem of gravitational waves detecting which description is possible with the help of the Einstein's equation of general relativity is solved. The further increase of sensitivity of a measuring method will allow estimating more adequately correctness and other alternative theories of gravitation [5].

From the physical point of view the general relativity theory assumes that the mass curvature is a space-time [6]. This curvature of space-time influences all particles moving in space, including and what creates a curvature. Influence is carried out and on massless particles, for example-photons. It is connected to a curvature of geodetic lines of space-time on which photons move. Space-time curvature in the general relativity theory identifies with occurrence of some gravitational fields due to which there is an interaction of mass particles. Photons also change the frequency in a gravitational field.

Gravitational waves are propagating oscillations of the curved space-time as similar as the waves on a water surface are propagating oscillations of the water particles.

However in the submitted physical picture of gravitation there is no the major element-quantization of a gravitational field.

Attempts to solve a problem of the gravitational waves quantization with the help of 5-dimensional space-time use [7] can hardly lead to success. Apparently, the theory of 5-dimensional space-time now has only a historical value. By comparison of distance from the source of gravitational waves calculated by the attenuation of experimentally registered gravitational waves and by the red displacement of electromagnetic radiation it has been established [8] that dimension of our space-time is equal $\sim 4 \pm 0.1$. Thus our space-time is described by four coordinates: time and three spatial coordinates.

Assuming as a whole correctness of the Einstein's equation for a gravitational field we research some features of a gravitational radiation and quantization of the gravitational waves.

\section{Photon in Constant Homogeneous Gravitational Field}

For the beginning we shall consider how frequency of an electromagnetic radiation quantum (photon) in a constant homogeneous gravitational field changes. Research we shall carry out in flat space-time the name Minkowski's space. The interval in inertial reference system looks like [6]:

$$
\begin{aligned}
\mathrm{d} s^{2} & =c^{2} \mathrm{~d} \tau^{2}-\mathrm{d} X^{2}-\mathrm{d} Y^{2}-\mathrm{d} Z^{2} \\
& =g_{00}\left(\mathrm{~d} X^{0}\right)^{2}-g_{11}\left(\mathrm{~d} X^{1}\right)^{2}-g_{22}\left(\mathrm{~d} X^{2}\right)^{2}-g_{33}\left(\mathrm{~d} X^{3}\right)^{2}
\end{aligned}
$$

where designations there are $X=X^{1}, Y=X^{2}, Z=X^{3}$-the Cartesian coordinates, $c$-a light velocity in vacuum, $\mathrm{d} \tau$-an interval proper time between events [6], so $c \mathrm{~d} \tau=\mathrm{d} X^{0}, g_{00}=1, g_{11}=g_{22}=g_{33}=-1$-the metric tensor components which signature $(+,-,-,-)$.

From (1) follows: 


$$
\mathrm{d} \tau=\frac{1}{c} \sqrt{g_{00}} \mathrm{~d} X^{0}
$$

As it will be shown further in a gravitational field $g_{00}<1$. Therefore, proper (or own) time flows that more slowly than is less $g_{00}$ in the given point of space ( $\Delta \tau=\tau_{2}-\tau_{1}$ decreases; $\tau_{2}$ in field it is less $\tau_{2}$ outside of field). The clock per a gravitational field is slow.

A function of Lagrange of a particle in the gravitational field looks like [6]:

$$
L=T-U=-m c^{2} \sqrt{1-\frac{V^{2}}{c^{2}}}-m \varphi_{g}=-m c^{2}\left(1-\frac{1}{2} \frac{V^{2}}{c^{2}}\right)-m \varphi_{g}
$$

where $m$ there is mass of a particle in a field, $V$-its velocity, $\varphi_{g}$ - gravitational potential of a field, so acceleration $\dot{\boldsymbol{V}}=-\operatorname{grad} \varphi_{g}$.

Action for a particle in a gravitational field is equal [6]:

$$
S=\int L \mathrm{~d} \tau=-m c \int\left(c-\frac{V^{2}}{2 c}+\frac{\varphi_{g}}{c}\right) \mathrm{d} \tau=-m c \int \mathrm{d} s
$$

where $S$ there is the action for a free material particle, $\mathrm{d} s$-interval.

Hence, the square of the interval $\mathrm{d} s$ is equal:

$$
\begin{aligned}
\mathrm{d} s^{2} & =\left(c-\frac{V^{2}}{2 c}+\frac{\varphi_{g}}{c}\right)^{2} \mathrm{~d} \tau^{2}=\left(c^{2}-V^{2}+\frac{\varphi_{g}^{2}}{c^{2}}+2 \varphi_{g}\right) \mathrm{d} \tau^{2} \\
& =c^{2}\left(1+2 \frac{\varphi_{g}}{c^{2}}+\frac{\varphi_{g}^{2}}{c^{4}}\right) \mathrm{d} \tau^{2}-\mathrm{d} \boldsymbol{r}^{2}=c^{2}\left(1+\frac{\varphi_{g}}{c^{2}}\right)^{2} \mathrm{~d} \tau^{2}-\mathrm{d} \boldsymbol{r}^{2}
\end{aligned}
$$

where it is used $\mathrm{d} \boldsymbol{r}=\boldsymbol{V} \mathrm{d} \tau$.

Thus according to (1) the component of metric tensor $g_{00}$ in a gravitational field decreases:

$$
g_{00}=\left(1+\frac{\varphi_{g}}{c^{2}}\right)^{2} \text { and } \sqrt{g_{00}}=1+\frac{\varphi_{g}}{c^{2}}
$$

where $\varphi_{g}$ there is negative size.

For the further analysis we use concept of an eikonal [6]. Eikonal there is a phase of the periodic function describing a field of electromagnetic wave:

$$
\phi=k \boldsymbol{q}-\delta \tau
$$

where $\boldsymbol{k}$ there is a wave vector of an eikonal, $\boldsymbol{q}$-a coordinate vector of an eikonal (it is optional Cartesian), $\delta$-cyclic frequency of the eikonal.

Taking into account (2) and (7) it is possible to find the eikonal frequency (a photon frequency in the given point in a proper time):

$$
\delta=-\frac{\partial \phi}{\partial \tau}=-\frac{\partial \phi}{\partial X^{0}} \frac{\partial X^{0}}{\partial \tau}=-\frac{c}{\sqrt{g_{00}}} \frac{\partial \phi}{\partial X^{0}}
$$

If to use the world time $t$ (outside of a gravitational field), so that $t=\frac{X^{0}}{c}$ the photon cyclic frequency measured in world time is equal $\delta_{0}=-\frac{\partial \phi}{\partial t}=-c \frac{\partial \phi}{\partial X^{0}}$. 
Hence, according to (8) with the account (6) we have:

$$
\delta=\frac{\delta_{0}}{\sqrt{g_{00}}}=\frac{\delta_{0}}{1+\frac{\varphi_{g}}{c^{2}}}
$$

where $\delta_{0}$ there is photon frequency at absence of a gravitational field.

Thus, photon frequency depends on size of a gravitational field potential. As the gravitational field potential it is negative size at approach to the creating a field bodies, the photon frequency $\delta$ grows, and at removal falls (red displacement). For example, for a body in mass $M$ the potential of a field depends on radius $r$ under the formula $\varphi_{g}=-k \frac{M}{r}$.

The eikonal wave vector (or a photon wave vector) $\boldsymbol{k}=\frac{\partial \phi}{\partial \boldsymbol{q}}$, and 4-impulse in the Cartesian coordinates is equal $k_{i}=-\frac{\partial \phi}{\partial X_{i}}$. But for the 4-impulse the formula $k_{i} k^{i}=k^{0} k^{0}-\boldsymbol{k} \boldsymbol{k}=0$ is correct. Hence, $\frac{\partial \phi}{\partial X_{i}} \frac{\partial \phi}{\partial X^{i}}=0$ there is the eikonal equation.

Let's note that the eikonal is a quantized size. The eikonal quantum is equal:

$$
S_{0}=\hbar \phi
$$

where $\hbar$ there is reduced Planck's constant.

\section{Generating of a Gravitational Radiation, and Gravitational Waves}

Gravitational radiation arises at the relative movement of massive bodies. We shall consider the weak gravitational radiation created by the rather small bodies which move with small velocities.

Let's assume that gravitational waves are connected to small change of a metric tensor. In this case it is possible to write down [6]:

$$
g_{i k}=g_{i k}^{(0)}+h_{i k}
$$

where $g_{i k}^{(0)}$ there is basic part of a metric tensor created by a body, $h_{i k}$-the small gravitational additive of a metric tensor concerning to the radiated waves, so $g_{i k}^{(0)} \gg h_{i k}$.

The gravitational field is described by the Einstein's equation. For writing of the Einstein's equation it is necessary, first of all, by the mathematical to describe a curvature of space-time. For the description of the mathematical curvature of space-time it is used a tensor of curvature (Riemann's tensor) [6]:

$$
R_{i k l m}=\left(\frac{\partial \Gamma_{k m}^{i}}{\partial X^{l}}-\frac{\partial \Gamma_{k l}^{i}}{\partial X^{m}}\right)+\left(\Gamma_{n l}^{i} \Gamma_{k m}^{n}-\Gamma_{n m}^{i} \Gamma_{k l}^{n}\right)
$$

where $\Gamma_{k l}^{i}$ there is a projection of a derivative unit vector $\boldsymbol{e}_{k}$ on coordinate $X^{l}$ on a coordinate axis $X^{i}$-Cristoffel's symbol $\Gamma_{k l}^{i} e_{i}=\frac{\partial e_{k}}{\partial X^{l}}$. Cristoffel's 
symbols it is the functions of coordinates characterizing change a component of a vector at its parallel displacement. All indexes, bottom (covariant, usually functional sizes) and top (contravariant, usually coordinate sizes) accept values 0 (a time index), 1, 2, 3 (coordinate indexes). As it is usual summation is carried out on indexes repeating in products.

Cristoffel's symbols can be expressed through metric tensor [6]:

$$
\Gamma_{k l}^{i}=\frac{1}{2} g^{i m}\left(\frac{\partial g_{m k}}{\partial X^{l}}+\frac{\partial g_{m l}}{\partial X^{k}}-\frac{\partial g_{k l}}{\partial X^{m}}\right)
$$

Thus curvature tensor of a space-time it is determined by a velocity and rapidly of a metric tensor $g_{i k}$ change in the space-time. Generally there are 10 components of a metric tensor: 4 - with identical indexes $(00,11,22,33)$, and 6 with different indexes $\left(01,02,03,12,13,23, C_{4}^{2}=\frac{4 \times 3}{1 \times 2}=6\right)$.

The curvature tensor is a fourth rank. Physically the curvature of space-time can be described only the second rank tensor since energy-impulse tensor creating this curvature is the second rank tensor. Therefore we shall pass with the help of a curtailing operation in (12) to the second rank tensor (Ricci's tensor):

$$
R_{i k}=g^{l m} R_{l i m k}=\left(\frac{\partial \Gamma_{i k}^{l}}{\partial X^{l}}-\frac{\partial \Gamma_{i l}^{l}}{\partial X^{k}}\right)+\left(\Gamma_{i k}^{l} \Gamma_{l m}^{m}-\Gamma_{i l}^{m} \Gamma_{k m}^{l}\right)
$$

The Ricci's tensor it is symmetrical:

$$
R_{i k}=R_{k i}
$$

Further we shall introduce a scalar curvature of a space-time under the formula:

$$
R=g^{i k} R_{i k}
$$

where $g^{i k}$ there is contravariant metric tensor.

Using the Ricci's tensor (14), a scalar curvature of a space-time (16), and also a metric tensor $g_{i k}$, the Einstein has written down the basic equation for a gravitational field:

$$
R_{i k}-\frac{1}{2} g_{i k} R=\frac{8 \pi k}{c^{4}} T_{i k}
$$

The left part of this equation refers to Einstein's tensor $E_{i k}=R_{i k}-\frac{1}{2} g_{i k} R$. It characterizes geometrical properties of a space-time in particular its curvature. The right part of the equation includes an energy-impulse tensor of the second rank describing the source which creates the curvature of a space-time:

$$
T_{i k}=\left(\begin{array}{llll}
T_{00} & T_{01} & T_{02} & T_{03} \\
T_{10} & T_{11} & T_{12} & T_{13} \\
T_{20} & T_{21} & T_{22} & T_{23} \\
T_{30} & T_{31} & T_{32} & T_{33}
\end{array}\right)
$$

Einstein's Equation (17) can be written down in the other kind. We shall multiply at left the Equation (17) on the contravariant metric tensor $g^{i k}$ : 


$$
g^{i k} R_{i k}-\frac{1}{2} g^{i k} g_{i k} R=\frac{8 \pi k}{c^{4}} g^{i k} T_{i k}
$$

Taking into account $R=g^{i k} R_{i k}$ and $g^{i k} g_{i k}=4$ (in a four-dimensional space-time) we shall find:

$$
-R=\frac{8 \pi k}{c^{4}} \operatorname{Sp} T_{i k}=\frac{8 \pi k}{c^{4}} T
$$

where it is designated $\operatorname{Sp} T_{i k}=T$.

Having substituted (20) in the Einstein's Equation (17) we shall find:

$$
R_{i k}=\frac{8 \pi k}{c^{4}}\left(T_{i k}-\frac{1}{2} g_{i k} T\right)
$$

Outside of a spherical symmetric body in mass $M$ creating a gravitational field the energy-impulse tensor is equal to zero $T_{i k}=T=0$. In this case according to (21):

$$
R_{i k}=0
$$

Zero size of the Ricci's tensor according to the Equation (22) does not mean that there is no curvature of a space-time. The space-time curvature which is characterized by the Riemann's tensor (12) is kept in distance from the bodies creating this curvature.

Let's substitute the Cristoffel's symbols (13) in the first bracket of a Riemann's curvature tensor (12) of space-time. In result we have:

$$
R_{i k l m}=\frac{1}{2}\left(\frac{\partial^{2} g_{i m}}{\partial X^{k} \partial X^{l}}+\frac{\partial^{2} g_{k l}}{\partial X^{i} \partial X^{m}}-\frac{\partial^{2} g_{k m}}{\partial X^{i} \partial X^{l}}-\frac{\partial^{2} g_{i l}}{\partial X^{k} \partial X^{m}}\right)+g_{n p}\left(\Gamma_{k l}^{n} \Gamma_{i m}^{p}-\Gamma_{k m}^{n} \Gamma_{i l}^{p}\right)
$$

In the Formula (23) we shall substitute (11), and neglecting for the small gravitational additive $h_{i k}$ the products of the Cristoffel's symbols we shall find:

$$
R_{i k l m}=\frac{1}{2}\left(\frac{\partial^{2} h_{i m}}{\partial X^{k} \partial X^{l}}+\frac{\partial^{2} h_{k l}}{\partial X^{i} \partial X^{m}}-\frac{\partial^{2} h_{k m}}{\partial X^{i} \partial X^{l}}-\frac{\partial^{2} h_{i l}}{\partial X^{k} \partial X^{m}}\right)
$$

Let's pass to the Ricci's tensor multiplying (24) at the left on not perturbed metric tensor $g^{(0) l m}$. Taking into account:

$$
g_{i k} g^{i k}=\left(g_{i k}^{(0)}+h_{i k}\right)\left(g^{(0) i k}-h^{i k}\right)=g_{i k}^{(0)} g^{(0) i k}+h_{i k} g^{(0) i k}-g_{i k}^{(0)} h^{i k}-h_{i k} h^{i k} \approx g_{i k}^{(0)} g^{(0) i k}
$$

for contravariant components as against (11) it is possible to write down approximately (product of additives to metric tensor it is neglected):

$$
g^{l m}=g^{(0) l m}-h^{l m}
$$

Thus we shall find:

$$
R_{i k}=\frac{1}{2}\left(-g^{(0) l m} \frac{\partial^{2} h_{i k}}{\partial X^{l} \partial X^{m}}+\frac{\partial^{2} h_{i}^{l}}{\partial X^{k} \partial X^{l}}+\frac{\partial^{2} h_{k}^{l}}{\partial X^{i} \partial X^{l}}-\frac{\partial^{2} h}{\partial X^{i} \partial X^{k}}\right)
$$

where it is designated $h=h_{i}^{i}$. Rise of indexes was carried out by a rule $h_{i}^{k}=g^{(0) k l} h_{i l}$.

We use in (20) the additional gauge condition [9]: 


$$
\frac{\partial^{2} h_{i}^{l}}{\partial X^{k} \partial X^{l}}+\frac{\partial^{2} h_{k}^{l}}{\partial X^{i} \partial X^{l}}=\frac{\partial^{2} h}{\partial X^{i} \partial X^{k}}
$$

Let's show that a condition (27) is gauge condition. We shall transform the left part:

$$
\frac{\partial^{2} h_{i}^{l}}{\partial X^{k} \partial X^{l}}+\frac{\partial^{2} h_{k}^{l}}{\partial X^{i} \partial X^{l}}=\frac{\partial}{\partial X^{l}}\left(\frac{\partial h_{i}^{l}}{\partial X^{k}}+\frac{\partial h_{k}^{l}}{\partial X^{i}}\right)=2 \frac{\partial}{\partial X^{l}}\left(\frac{\partial h_{i}^{l}}{\partial X^{k}}\right)=2 \frac{\partial}{\partial X^{k}}\left(\frac{\partial h_{i}^{l}}{\partial X^{l}}\right)
$$

Equating (27) and (28), we shall find:

$$
\frac{\partial h}{\partial X^{i}}=2 \frac{\partial h_{i}^{l}}{\partial X^{l}}+\text { const }
$$

If scalar curvature of space due to the gravitational additive of metric tensor is small $h \rightarrow 0$, and accepting a constant of integration equal to zero that is reached by a choice of the reference system beginning, we receive:

$$
\frac{\partial h_{i}^{l}}{\partial X^{l}}=\frac{\partial h_{i}^{0}}{\partial c t}+\frac{\partial h_{i}^{1}}{\partial X^{1}}+\frac{\partial h_{i}^{2}}{\partial X^{2}}+\frac{\partial h_{i}^{3}}{\partial X^{3}}=\frac{\partial h_{i}^{0}}{\partial c t}+\operatorname{div} \boldsymbol{h}_{i}=0
$$

The received condition is similar to Lorentz's gauge in electrodynamics. Therefore equality (27) is a gauge condition.

Thus Ricci's tensor (26) with the account (27) looks like:

$$
R_{i k}=\frac{1}{2}\left(-g^{(0) l m} \frac{\partial^{2} h_{i k}}{\partial X^{l} \partial X^{m}}\right)=\frac{1}{2}\left(\frac{\partial^{2} h_{i k}}{\partial X_{\alpha} \partial X_{\alpha}}-\frac{1}{c^{2}} \frac{\partial^{2} h_{i k}}{\partial t^{2}}\right)
$$

Let's substitute the received expression of the Ricci's tensor (31) in Einstein's equation for a gravitational field as (21). In empty space outside the mass creating a gravitational field we shall assume the diagonal components of energy-impulse tensor equal to zero $T=0$. In this case we have:

$$
\frac{\partial^{2} h_{i k}}{\partial X_{\alpha} \partial X_{\alpha}}-\frac{1}{c^{2}} \frac{\partial^{2} h_{i k}}{\partial t^{2}}=\frac{16 \pi k}{c^{4}} T_{i k}
$$

The solution of the Equation (32) is well-known from the theory of retarded potentials [10]. At small speeds of movement of a gravitational radiation source:

$$
h_{i k}=-\frac{4 k}{c^{4} r_{0}} \int_{V} T_{i k}\left(t-\frac{r_{0}}{c}\right) \mathrm{d} V
$$

where $r_{0}$ there is distance from the mass center of a field source up to a point of supervision, $V$ - volume on which integration is carried out.

Let's find a derivative of the left and right parts of the Equation (31) on $X_{k}$ :

$$
\frac{\partial R_{i k}}{\partial X_{k}}=\frac{1}{2}\left(-g^{(0) l m} \frac{\partial^{2}}{\partial X^{l} \partial X^{m}}\left(\frac{\partial h_{i k}}{\partial X_{k}}\right)\right)=\frac{8 \pi k}{c^{4}} \frac{\partial T_{i k}}{\partial X_{k}}
$$

Taking into account (30) at replacement of an index $l$ on an index $k$, we have:

$$
\frac{\partial T_{i k}}{\partial X_{k}}=0
$$

For simplification of transformations have assumed $h \rightarrow 0$, i.e. almost flat space-time. 
Let's write down the Equation (35) having allocated a time component:

$$
\frac{\partial T_{\alpha \gamma}}{\partial X^{\gamma}}-\frac{\partial T_{\alpha 0}}{\partial X^{0}}=0 \text { and } \frac{\partial T_{0 \gamma}}{\partial X^{\gamma}}-\frac{\partial T_{00}}{\partial X^{0}}=0
$$

Let's multiply the first Equation (36) on $X^{\beta}$ and we shall integrate it on all space:

$$
\frac{\partial}{\partial X^{0}} \int T_{\alpha 0} X^{\beta} \mathrm{d} V=\int \frac{\partial T_{\alpha \gamma}}{\partial X^{\gamma}} X^{\beta} \mathrm{d} V=\int \frac{\partial\left(T_{\alpha \gamma} X^{\beta}\right)}{\partial X^{\gamma}} \mathrm{d} V-\int T_{\alpha \beta} \mathrm{d} V=-\int T_{\alpha \beta} \mathrm{d} V
$$

where according to Gauss's theorem $\int \frac{\partial\left(T_{\alpha \gamma} X^{\beta}\right)}{\partial X^{\gamma}} \mathrm{d} V=\oint\left(T_{\alpha \gamma} X^{\beta}\right) \mathrm{d} S=0$ for $T_{\alpha \gamma}=0$ on infinity.

The equality (37) can be copied in a symmetrical kind:

$$
\int T_{\alpha \beta} \mathrm{d} V=-\frac{1}{2} \frac{\partial}{\partial X^{0}} \int\left(T_{\alpha 0} X^{\beta}+T_{\beta 0} X^{\alpha}\right) \mathrm{d} V
$$

Similarly we shall multiply the second Equation (36) on $X^{\alpha} X^{\beta}$ and we shall integrate it on all space:

$$
\begin{aligned}
& \frac{\partial}{\partial X^{0}} \int T_{00} X^{\alpha} X^{\beta} \mathrm{d} V=\int \frac{\partial T_{0 \gamma}}{\partial X^{\gamma}} X^{\alpha} X^{\beta} \mathrm{d} V \\
& =\int \frac{\partial\left(T_{0 \gamma} X^{\alpha} X^{\beta}\right)}{\partial X^{\gamma}} \mathrm{d} V-\int T_{\alpha 0} X^{\beta} \mathrm{d} V-\int T_{\beta 0} X^{\alpha} \mathrm{d} V
\end{aligned}
$$

From (39) follows:

$$
\frac{\partial}{\partial X^{0}} \int T_{00} X^{\alpha} X^{\beta} \mathrm{d} V=-\int\left(T_{\alpha 0} X^{\beta}+T_{\beta 0} X^{\alpha}\right) \mathrm{d} V
$$

Substituting (40) in (38) we find:

$$
\int T_{\alpha \beta} \mathrm{d} V=\frac{1}{2} \frac{\partial^{2}}{\partial X^{02}} \int T_{00} X^{\alpha} X^{\beta} \mathrm{d} V
$$

Taking into account (41), $T_{00}=\rho c^{2}$, and $X_{0}=c t$ the Formula (33) can be written down:

$$
h_{i k}=-\frac{2 k}{c^{4} r_{0}} \frac{\partial^{2}}{\partial t^{2}} \int \rho X^{i} X^{k} \mathrm{~d} V
$$

Let's expand into series the gravitational potential [6]:

$$
\varphi=\varphi_{0}+\varphi_{1}+\varphi_{2}+\cdots=-k\left(\frac{M}{r_{0}}+\frac{1}{6} D_{i k} \frac{\partial^{2}}{\partial X_{i} \partial X_{k}} \frac{1}{r_{0}}+\cdots\right)
$$

where dipole component of potential $\varphi_{1}=0$ owing to a choice of a reference system beginning in the mass center of system, $D_{i k}=\int \rho\left(3 X^{i} X^{k}-X^{i 2}\right) \mathrm{d} V$ there is quadrupole moment of system. On the big distances from system it is possible to write down $D_{i k} \approx \int 3 \rho X^{i} X^{k} \mathrm{~d} V$.

Thus the Formula (42) can be written down as:

$$
h_{i k}=-\frac{2 k}{3 c^{4} r_{0}} \frac{\partial^{2} D_{i k}}{\partial t^{2}}
$$


where $i, k=1,2,3$.

The energy flux of gravitational radiation in the given direction is equal [9]:

$$
-\frac{\mathrm{d} \varepsilon}{\mathrm{d} t}=\frac{c^{3}}{16 \pi k} \sum_{i, k}\left(\frac{\partial h_{i k}}{\partial t}\right)^{2}=\frac{c^{3}}{16 \pi k}\left(\frac{2 k}{3 c^{4} r_{0}}\right)^{2} \sum_{i, k}\left(\frac{\partial^{3} D_{i k}}{\partial t^{3}}\right)^{2}=\frac{k}{36 \pi c^{5} r_{0}^{2}} \sum_{i, k}\left(\frac{\partial^{3} D_{i k}}{\partial t^{3}}\right)^{2}
$$

Finding the energy flux of a gravitational radiation in the element of a solid angle $d \Omega$, and averaging it on all directions we shall receive [6]:

$$
-\frac{\mathrm{d} E}{\mathrm{~d} t}=\frac{k}{36 \pi c^{5} r_{0}^{2}} \int \frac{1}{5} \sum_{i, k}\left(\frac{\partial^{3} D_{i k}}{\partial t^{3}}\right)^{2} r_{0}^{2} \mathrm{~d} \Omega=\frac{k}{45 c^{5}} \sum_{i, k}\left(\frac{\partial^{3} D_{i k}}{\partial t^{3}}\right)^{2}
$$

\section{Gravitational Radiation of Double Stars}

Let's find the general energy flux of a gravitational radiation from the two bodies mass $m_{1}$ and $m_{2}$ the distance between which is equal $l$, moving on circular orbits with frequency of rotation $\delta$ around of the common mass center, Figure 1. Such system is characteristic for double stars.

As well as everyone symmetric tensor the tensor $D_{i k}$ can be reduced in the main axes.

Entering the reduced mass of system $\mu=\frac{m_{1} m_{2}}{m_{1}+m_{2}}$ we shall write down the quadrupole moments concerning axes $X, Y$, and $Z$ :

$$
\begin{gathered}
D_{X X}=\mu l^{2}\left(3 \cos ^{2} \delta t-1\right) \\
D_{Y Y}=\mu l^{2}\left(3 \sin ^{2} \delta t-1\right) \\
D_{Z Z}=-\mu l^{2}
\end{gathered}
$$

The quadrupole moments (47)-(49) satisfy to a condition [6]:

$$
D_{X X}+D_{Y Y}+D_{Z Z}=0
$$

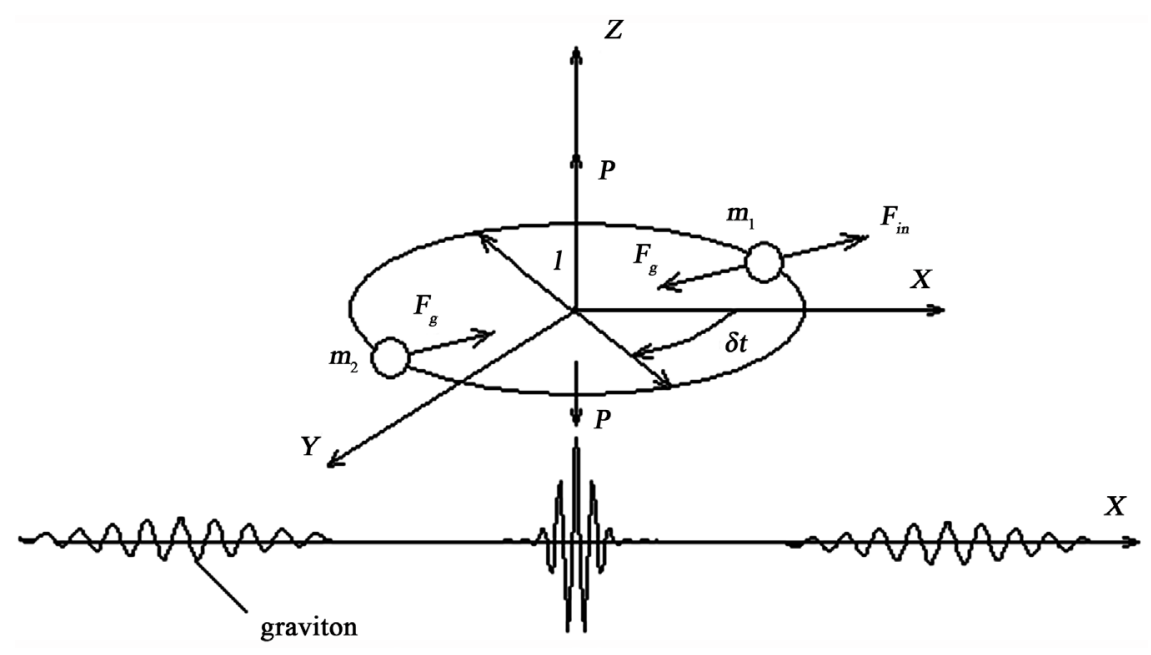

Figure 1. The scheme of bodies as the double star radiating gravitational waves and graviton flying in the field of a double star. 
According to the Formula (46) it is necessary to summarize the peak values of squares of third derivative on time from the quadrupole moments. It is connected by that in the Formula (33) the peak values of metric tensor $h_{i k}$ are used. Carrying out simple transformations we find:

$$
\begin{aligned}
\sum_{i, k}\left(\frac{\partial^{3} D_{i k}}{\partial t^{3}}\right)^{2} & =\left(\frac{\partial^{3} D_{X X}}{\partial t^{3}}\right)^{2}+\left(\frac{\partial^{3} D_{Y Y}}{\partial t^{3}}\right)^{2}+\left(\frac{\partial^{3} D_{Z Z}}{\partial t^{3}}\right)^{2} \\
& =2\left(12 \mu l^{2} \delta^{3}\right)^{2}=288 \mu^{2} l^{4} \delta^{6}
\end{aligned}
$$

The derivative from the quadrupole moment $D_{Z Z}$ is equal to zero since it does not depend on time.

Substituting (51) in (46) we find:

$$
-\frac{\mathrm{d} E}{\mathrm{~d} t}=\frac{32}{5} \frac{k}{c^{5}}\left(\frac{m_{1} m_{2}}{m_{1}+m_{2}}\right)^{2} l^{4} \delta^{6}
$$

Let's note that the gravitational radiation energy flux

$P=\frac{1}{2}\left|\frac{\mathrm{d} E}{\mathrm{~d} t}\right|=\frac{16}{5} \frac{k}{c^{5}}\left(\frac{m_{1} m_{2}}{m_{1}+m_{2}}\right)^{2} l^{4} \delta^{6}$ goes in both directions along axis $Z$ [11], Figure 1.

The Formula (52) is frequently written in the other kind [12]. Using equivalence of gravitational and inert masses, and hence the forces $F_{g}=F_{i n}$, Figure 1, we receive $k\left(m_{1}+m_{2}\right)=\delta^{2} l^{3}$. Substituting the frequency $\delta$ in (52) we find:

$$
-\frac{\mathrm{d} E}{\mathrm{~d} t}=\frac{32}{5} \frac{k^{4}}{c^{5}} \frac{\left(m_{1} m_{2}\right)^{2}\left(m_{1}+m_{2}\right)}{l^{5}}
$$

Formulas (52) and (53) have absolutely classical (not quantum) character.

Outside a radiator of gravitational radiation the energy-impulse tensor $T_{i k}=0$. In this case the Equation (32) will be transformed to a kind:

$$
\frac{\partial^{2} h_{i k}}{\partial X_{\alpha} \partial X_{\alpha}}-\frac{1}{c^{2}} \frac{\partial^{2} h_{i k}}{\partial t^{2}}=0
$$

The Equation (45) is the wave equation reflecting a propagation of the gravitational waves in empty space. Taking into account the gauge condition (30) and the Equation (54) by analogy to electromagnetic waves is possible to draw a conclusion that gravitational waves are the cross-section waves propagating with a light velocity $c$.

The solution of the Equation (54) can be written down as [12]:

$$
h_{i k}=a e_{i k} \cos (\boldsymbol{r} \boldsymbol{X}-\delta t)
$$

where $a$ there is an amplitude of a wave, $\delta$-a frequency of gravitational waves, $\boldsymbol{r}$-a wave vector in a direction of a wave propagation. The value $e_{i k}$ there is a unit polarization tensor, obeying the conditions:

$$
e_{i k}=e_{k i}, \quad e_{i i}=0, \quad k_{i} e_{i k}=0, e_{i k} e_{i k}=1
$$

As against an electromagnetic wave which polarization is determined by a vector of the electric field oscillations, the polarization of a gravitational wave 
has essentially tensor character.

The third condition (56) is a condition of the gravitational waves cross-section.

\section{Quantization of Gravitational Waves}

At first point of view the quantization of a gravitational field can be carried out on analogies to a standard method of an electromagnetic field quantization, in particular, by a method of secondary quantization. Really, the equations of an electromagnetic wave include the wave equation and gauge equation. Similarly, the equation of a gravitational wave (54) is accompanied by the gauge Equation (27) which for a weak gravitational field passes in Lorentz's gauge (30) identical to one gauges used in electrodynamics.

Therefore the gravitational field is a version of gauge fields [13].

However, for a gravitational field the situation is complicated two problems. First, the physical mechanism of reflection of gravitational waves from physical structures is not clear. Second, it is not known whether origination, and how of standing gravitational waves is possible.

\subsection{Action of the Systems Gravitational Field-Particle}

Both these unresolved problems now do application of a standard method for quantization of gravitational waves problematic.

Quantization of electromagnetic waves was carried out by quantization of the volumetric density of action in space of the generalized coordinates:

$$
s=\int l \mathrm{~d} t=\int(T-U) \mathrm{d} t=\int T \mathrm{~d} t-\int U \mathrm{~d} t
$$

where $l=T-U$ there is total Lagrangian of a system a photon-particle and their interaction [14].

Let's consider Lagrangian 1 of a systems a gravitational field-particle. Lagrangian of this system looks like:

$$
l=T-U=\left(\rho-\rho_{0}\right) c^{2} \sqrt{-g}-\left(\rho \varphi_{g}-l_{g}\right) \sqrt{-g}
$$

where $T=\left(\rho-\rho_{0}\right) c^{2} \sqrt{-g}$ there is the volumetric density of a kinetic energy of a particle in a gravitational field, $U=\left(\rho \varphi_{g}-l_{g}\right) \sqrt{-g}$ - volumetric density of a potential energy of a particle in a field (energy of interaction of a particle and field) including Lagrangian of the field [6]:

$$
l_{g}=-\frac{c^{4}}{16 \pi k} R
$$

where $R$ there is a scalar curvature of a space-time, $g<0$-the determinant of a metric tensor, $\sqrt{-g}$-defines the dependence of an volume normalizing element on space curvature, $\rho$-mass density of a particle in a field, $\rho_{0}$-mass density of a rest particle, $\varphi_{g}$-gravitational potential of a field.

Lagrangian to the Lagrange's equation submits which looks like:

$$
\frac{\mathrm{d}}{\mathrm{d} t}\left(\frac{\partial l}{\partial \dot{\boldsymbol{q}}}\right)=\frac{\partial l}{\partial \boldsymbol{q}}
$$


For the generalized velocity we use size:

$$
\dot{\boldsymbol{q}}=\sqrt{2 T}
$$

In this case the known formula for volumetric density of energy [6] is correct:

$$
w=\dot{\boldsymbol{q}} \frac{\partial l}{\partial \dot{\boldsymbol{q}}}-l=\sqrt{2 T} \frac{\partial l}{\partial \sqrt{2 T}}-T+U=\sqrt{T} \frac{\partial l}{\partial T} \frac{\partial T}{\partial \sqrt{T}}-T+U=T+U
$$

owing to $\frac{\partial l}{\partial T}=1$.

From Formulas (58) and (61) follows that kinetic and potential energy of a particle in a gravitational field are equal:

$$
T=\frac{\dot{\boldsymbol{q}}^{2}}{2}=\left(\rho-\rho_{0}\right) c^{2} \sqrt{-g}, \quad U=\left(\rho \varphi_{g}-l_{g}\right) \sqrt{-g}
$$

Thus both components of volumetric density of action depend on a body density which creates curvature of a space-time.

Therefore in the Einstein's Equation (17) the right part of the equation dependent on mass creating a gravitational field should be subject to quantization only. The time is not quantized value. Apparently are not quantized all parameters of a space-time (scalar curvature, metric tensor, Ricci's tensor, etc.). To quantization can be subject to only energy-impulse tensor.

\subsection{Graviton Energy and Quantum Gravitational Eikonal}

The quantum of a gravitational field-a graviton arises far from massive bodies where there is no gravitational field of these bodies. It arises due to curving by graviton of the Riemann's spaces. Therefore a graviton has mass. The graviton mass is equal:

$$
m=\frac{E}{c^{2}}
$$

where $E$ there is a graviton energy, $c$-a light velocity.

Trace $T$ an energy-impulse tensor in Einstein's equation is connected to scalar curvature of space-time $R$ a ratio (20).

We use a sign plus in the Formula (20), since sizes in the left and right parts of equality positive. A sign minus is necessary to use in Lobachevski's space to which Einstein's equation it is applicable as absolutely general law of the nature.

If the graviton is propagated in a direction of an axis $X_{1}$ the diagonal components of a metric tensor owing to the cross-section of gravitational waves following: $h_{11}=0, h_{22}=-h_{33}$ [11]. The same ratio of components should be according to (32) in the energy-impulse tensor of a graviton (18). Therefore a trace of a graviton energy-impulse tensor is equal

$$
T=T_{00}=\frac{m}{\mathrm{~V}} c^{2}=\frac{E}{\mathrm{~V}}
$$

where $\mathrm{V}$ there is normalizing volume. According to (20) the scalar curvature of space-time is equal: 


$$
R=\frac{8 \pi k}{c^{4}} \frac{E}{\mathrm{~V}}
$$

Action of a gravitational field is equal:

$$
S=\int l_{g} \mathrm{dVd} t
$$

Lagrangian of a gravitational field it is equal [6]:

$$
l_{g}=-\frac{c^{4}}{16 \pi k} R
$$

Substituting (67) in (66) and assuming approximately constancy of scalar curvature in area of a graviton we shall find:

$$
S=\int l_{g} \mathrm{dVd} t=-\frac{c^{4}}{16 \pi k} \mathrm{~V} R t+C
$$

where $C$ there is a constant of integration which can depend from $X_{1}$.

Substituting (65) in (68) we have:

$$
S=-\frac{1}{2} E t+C
$$

Further to similarly electromagnetic field, see (10), we shall enter into consideration concept of a quantum gravitational eikonal $S=\hbar \phi$ where $\hbar$ there is Planck's reduced constant, $\phi=r X_{1}-\omega t$-its phase, $r$-wave number of a graviton, $\omega$-its own frequency. We assume function of the quantum gravitational eikonal $S\left(X_{1}, t\right)$ approximately linear in a weak gravitational field [6] [15]. We shall note equivalence of quantum gravitational eikonal and actions of system a gravitational field-particle. Both sizes submit to a principle of a minimum (to Maupertuis' principle for action or Fermat's for eikonal) [6].

Let's equate a quantum gravitational eikonal and action:

$$
S=\hbar \phi=\hbar\left(r X_{1}-\omega t\right)=-\frac{1}{2} E t+C
$$

From the Formula (70) follows that graviton energy is equal:

$$
E=2 \hbar \omega
$$

and size $C=2 \hbar r X_{1}$. The spin of graviton it is $\pm 2 \hbar$.

Energy of a relativistic mass particle is equal $E=\sqrt{p^{2} c^{2}+m_{0}^{2} c^{4}}$. Therefore, the Formula (71) allows to assume that as against an ordinary particle the graviton rest mass is equal to zero $m_{0}=0$, and the impulse of the graviton is equal $p=\frac{E}{c}=\frac{2 \hbar \omega}{c}=2 \hbar r$.

Having substituted (71) in (64) we find $m=\frac{E}{c^{2}}=\frac{2 \hbar \omega}{c^{2}}$ hence the mass of a graviton is proportional to its frequency. If for a graviton (by analogy to a photon) the rule of "red displacement" operates then at removal from massive bodies the graviton frequency, and hence its mass should decrease down to disappearance of the graviton (gravitonic darkness ${ }^{1}$ ). At approach a graviton to the

${ }^{1}$ It is possible that in the Universe there are the vivid individuals seeing with the help of gravitons as well as Earth's individuals see with the help of photons. 
massive bodies the graviton frequency and its mass should increase.

If to accept for example the frequency of background thermal gravitational radiation $\omega=1.26 \times 10^{12} \mathrm{~s}^{-1}$ [11] then graviton energy is $E=2 \hbar \omega=2.66 \times 10^{-15} \mathrm{erg}=0.00166 \mathrm{eV}$.

A graviton mass (64) of the background thermal gravitational radiations $m=2.96 \times 10^{-26} \mathrm{~g}$.

Propagation of a graviton occurs in a direction of a normal to constant eikonal surface. Thus we have average radius of curvature of a constant eikonal surface (curvature of the Riemann's spaces) in the given approximation (a weak gravitational field) are much greater of a graviton wave lengths $\lambda=\frac{2 \pi c}{\omega}$ [15].

\subsection{The Quantum Form of an Energy-Impulse Tensor}

Before to pass to the gravitational waves quantization we shall be solve one task. We shall find a gravitational field created any gas in space.

Let the size $K=k_{1} k_{2}$ is the Gaussian curvature of a 3-dimensional surface, and $k_{1}=\frac{1}{\varsigma_{1}}$ and $k_{2}=\frac{1}{\varsigma_{2}}$ there are curvature in mutually perpendicular direc tions, $\varsigma_{1}$ and $\varsigma_{2}$-radiuses of curvature in these directions. Scalar curvature $R$ is connected with Gaussian curvature a simple ratio $\frac{1}{2} R=K \quad$ [6].

Hence the Equation (20) can be transformed to a kind:

$$
-\frac{2}{\varsigma_{1} \varsigma_{2}}=\frac{8 \pi k}{c^{4}}\left(T_{00}+T_{11}+T_{22}+T_{33}\right)
$$

If a body creating a gravitational field is a gas, $T_{11}=T_{22}=T_{33}=P$ there is pressure in gas, a size $T_{00}=\rho c^{2}, \rho$-a gas density. Neglecting pressure in gas in comparison with a mass component $T_{00}=\rho c^{2}$, we have:

$$
-\frac{1}{\varsigma_{1} \varsigma_{2}}=\frac{4 \pi k}{c^{4}} T_{00}=\frac{4 \pi k \rho}{c^{2}}
$$

Assuming $\varsigma_{1}=\varsigma_{2}$ we shall find:

$$
-\frac{1}{\varsigma_{1}^{2}}=\frac{4 \pi k \rho}{c^{2}}
$$

Using a constant $\frac{4 \pi k}{c^{2}}=0.93 \times 10^{-27} \mathrm{~cm} / \mathrm{g}$ and accepting gas density, for example, according to air density at a surface of the Earth $\rho=1.3 \times 10^{-3} \mathrm{~g} / \mathrm{cm}^{3}$, we find $\left|-\varsigma_{1}^{2}\right|=\frac{4 \pi k \rho}{c^{2}}=1.21 \times 10^{-30} 1 / \mathrm{cm}^{2}$. Hence the radius of space curvature is equal $\varsigma_{1}=\frac{1}{k_{1}}=0.91 \times 10^{15} \mathrm{~cm}=0.91 \times 10^{10} \mathrm{~km}$.

In calculation for reception of numerical values it was necessary to use the module of a square of curvature. There is an opportunity that space-time is a pseudosphere of Lobachevski's for which $K=-\frac{1}{\varsigma_{1}^{2}}$. But we do not examine this opportunity since the pseudosphere has an edge of return [16] it is difficult for 
them to compare any physical property of space-time.

We shall consider the energy-impulse tensor (18) in more detail. Into the energy-impulse tensor enters as making the stresses tensor:

$$
\sigma_{i k}=\left(\begin{array}{ccc}
T_{11} & T_{12} & T_{13} \\
T_{21} & T_{22} & T_{23} \\
T_{31} & T_{32} & T_{33}
\end{array}\right)=\left(\begin{array}{ccc}
\sigma_{X} & \tau_{X Y} & \tau_{X Z} \\
\tau_{Y X} & \sigma_{Y} & \tau_{Y Z} \\
\tau_{Z X} & \tau_{Z Y} & \sigma_{Z}
\end{array}\right)
$$

where $T_{11}, T_{22}, T_{33}$ there are normal stresses, other components $\sigma_{i k}=T_{i k}$ for $i \neq k$-tangential stresses. A component $T_{00}=\rho c^{2}$-volumetric density of energy, $T_{10}, T_{20}, T_{30}$-components of the impulse density multiplied on light velocity $\mathcal{c}$, $T_{01}, T_{02}, T_{03}$-components of the energy flux density divided on $c$.

In a basis of the further research we shall assume absence of a stresses state birth of empty space owing to its possible curvature.

In [17] it has been shown that in a fluid and gas there is an uncertainty of a sign on tangential stresses. Moreover the stresses tensor only approximately describes a stressed state of a fluid and gas. In a fluid and gas the stresses tensor is absent. For calculation of a fluid or gas flux it is necessary to use the vector formula connecting force $\mathrm{d} \boldsymbol{F}$ and velocity $\boldsymbol{V}$ as [17]:

$$
\mathrm{d} \boldsymbol{F}=\vec{\eta} \mathrm{d} \boldsymbol{S} \times \operatorname{rot} \boldsymbol{V}
$$

where $\mathrm{d} \boldsymbol{S}$ there is area of contacting layers in a fluid or gas, $\vec{\eta}$-viscosity tensor of the second rank, which diagonal components there are molecular viscosity, not diagonal components-turbulent viscosity.

If a flux of fluid or gas occurs for example in a direction axes $X$ the directions of vectors in the Formula (76) are shown on Figure 2.

For our purposes in the Formula (76) using cyclic frequency of a medium rotation $\omega=\frac{1}{2} \operatorname{rot} V \quad[18]$ it is convenient to write down as:

$$
\mathrm{d} \boldsymbol{F}=2 \vec{\eta} \mathrm{d} \boldsymbol{S} \times \boldsymbol{\omega}
$$

The scalar variant of the Formula (77) looks like:

$$
\begin{aligned}
& \mathrm{d} F_{X} \boldsymbol{i}+\mathrm{d} F_{Y} \boldsymbol{j}+\mathrm{d} F_{Z} \boldsymbol{k} \\
& =2 \eta\left\{\left(\mathrm{d} S_{Y} \omega_{Z}-\mathrm{d} S_{Z} \omega_{Y}\right) \boldsymbol{i}+\left(\mathrm{d} S_{Z} \omega_{X}-\mathrm{d} S_{X} \omega_{Z}\right) \boldsymbol{j}+\left(\mathrm{d} S_{X} \omega_{Y}-\mathrm{d} S_{Y} \omega_{X}\right) \boldsymbol{k}\right\}
\end{aligned}
$$

where $\boldsymbol{i}, \boldsymbol{j}, \boldsymbol{k}$ there are in this case unit vectors of 3 -dimensional space. Assuming space homogeneous we use a scalar variant viscosity tensor $\eta$.

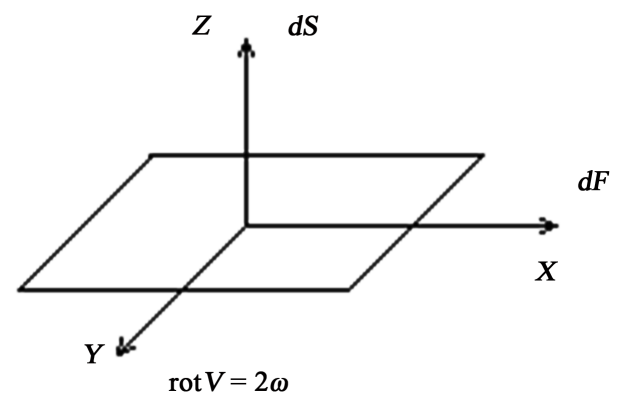

Figure 2. The directions of vectors in the Formula (76). 
At use of the Formula (76) the uncertainty of the tangential stresses sign and some other problems connected with stresses tensor [17] on which we shall not stop disappear.

We shall write down the energy-impulse tensor (18) in the following kind:

$$
T_{i k}=\left(\begin{array}{cccc}
\rho c^{2} & T_{01} & T_{02} & T_{03} \\
T_{10} & \frac{\mathrm{d} F_{1}}{\mathrm{~d} S_{1}} & \frac{\mathrm{d} F_{2}}{\mathrm{~d} S_{1}} & \frac{\mathrm{d} F_{3}}{\mathrm{~d} S_{1}} \\
T_{20} & \frac{\mathrm{d} F_{1}}{\mathrm{~d} S_{2}} & \frac{\mathrm{d} F_{2}}{\mathrm{~d} S_{2}} & \frac{\mathrm{d} F_{3}}{\mathrm{~d} S_{2}} \\
T_{30} & \frac{\mathrm{d} F_{1}}{\mathrm{~d} S_{3}} & \frac{\mathrm{d} F_{2}}{\mathrm{~d} S_{3}} & \frac{\mathrm{d} F_{3}}{\mathrm{~d} S_{3}}
\end{array}\right)
$$

where transition to digital indexes is carried out.

Taking into account (78), we have:

$$
T_{i k}=\left(\begin{array}{cccc}
\rho c^{2} & T_{01} & T_{02} & T_{03} \\
T_{10} & \frac{2 \eta\left(\mathrm{d} S_{2} \omega_{3}-\mathrm{d} S_{3} \omega_{2}\right)}{\mathrm{d} S_{1}} & \frac{2 \eta\left(\mathrm{d} S_{3} \omega_{1}-\mathrm{d} S_{1} \omega_{3}\right)}{\mathrm{d} S_{1}} & \frac{2 \eta\left(\mathrm{d} S_{1} \omega_{2}-\mathrm{d} S_{2} \omega_{1}\right)}{\mathrm{d} S_{1}} \\
T_{20} & \frac{2 \eta\left(\mathrm{d} S_{2} \omega_{3}-\mathrm{d} S_{3} \omega_{2}\right)}{\mathrm{d} S_{2}} & \frac{2 \eta\left(\mathrm{d} S_{3} \omega_{1}-\mathrm{d} S_{1} \omega_{3}\right)}{\mathrm{d} S_{2}} & \frac{2 \eta\left(\mathrm{d} S_{1} \omega_{2}-\mathrm{d} S_{2} \omega_{1}\right)}{\mathrm{d} S_{2}} \\
T_{30} & \frac{2 \eta\left(\mathrm{d} S_{2} \omega_{3}-\mathrm{d} S_{3} \omega_{2}\right)}{\mathrm{d} S_{3}} & \frac{2 \eta\left(\mathrm{d} S_{3} \omega_{1}-\mathrm{d} S_{1} \omega_{3}\right)}{\mathrm{d} S_{3}} & \frac{2 \eta\left(\mathrm{d} S_{1} \omega_{2}-\mathrm{d} S_{2} \omega_{1}\right)}{\mathrm{d} S_{3}}
\end{array}\right)
$$

The tensor (80) is an energy-impulse tensor creating a curvature of a space-time. For quantization of the energy-impulse tensor first of all the size $\eta$ should be expressed through the Planck's sizes: Planck's length $\left(\frac{\hbar k}{c^{3}}\right)^{\frac{1}{2}}$, Planck's time $\left(\frac{\hbar k}{c^{5}}\right)^{\frac{1}{2}}$ and Planck's mass $\left(\frac{\hbar c}{k}\right)^{\frac{1}{2}}$. Instead of size $\eta$ we use it Planck's value $\eta=\frac{\hbar}{\mathrm{V}}$ where $\mathrm{V}$ there is normalizing volume. Besides there are no reasons to assume the areas in (80) various therefore shall accept $\mathrm{d} S_{1}=\mathrm{d} S_{2}=\mathrm{d} S_{3}$. In this case the tensor (80) it will be transformed to a kind:

$$
T_{i k}=\left(\begin{array}{cccc}
\rho c^{2} & T_{01} & T_{02} & T_{03} \\
T_{10} & \frac{2 \hbar\left(\omega_{3}-\omega_{2}\right)}{\mathrm{V}} & \frac{2 \hbar\left(\omega_{1}-\omega_{3}\right)}{\mathrm{V}} & \frac{2 \hbar\left(\omega_{2}-\omega_{1}\right)}{\mathrm{V}} \\
T_{20} & \frac{2 \hbar\left(\omega_{3}-\omega_{2}\right)}{\mathrm{V}} & \frac{2 \hbar\left(\omega_{1}-\omega_{3}\right)}{\mathrm{V}} & \frac{2 \hbar\left(\omega_{2}-\omega_{1}\right)}{\mathrm{V}} \\
T_{30} & \frac{2 \hbar\left(\omega_{3}-\omega_{2}\right)}{\mathrm{V}} & \frac{2 \hbar\left(\omega_{1}-\omega_{3}\right)}{\mathrm{V}} & \frac{2 \hbar\left(\omega_{2}-\omega_{1}\right)}{\mathrm{V}}
\end{array}\right)
$$

Let's consider for example the energy-impulse tensor for propagation of gravitational radiation to a direction of axis $X_{1}$. Gravitational waves are cross-section hence the vector of frequency is directed along an axis $X_{1}$. In this case the Formula (81) looks like: 


$$
T_{i k}=\left(\begin{array}{cccc}
\rho c^{2} & T_{01} & T_{02} & T_{03} \\
T_{10} & 0 & \frac{2 \hbar \omega_{1}}{\mathrm{~V}} & -\frac{2 \hbar \omega_{1}}{\mathrm{~V}} \\
T_{20} & 0 & \frac{2 \hbar \omega_{1}}{\mathrm{~V}} & -\frac{2 \hbar \omega_{1}}{\mathrm{~V}} \\
T_{30} & 0 & \frac{2 \hbar \omega_{1}}{\mathrm{~V}} & -\frac{2 \hbar \omega_{1}}{\mathrm{~V}}
\end{array}\right)
$$

Asymmetry of the energy-impulse tensor $T_{i k}$ is connected with basic refusal from use symmetric stress tensor [17].

In the tensor of energy-impulse the components $\pm \frac{2 \hbar \omega_{1}}{\mathrm{~V}}= \pm \varepsilon$ enter which characterize volumetric density of energy of gravitational radiation quantum-graviton. Two signs of the spin are reflect two directions of polarization: (plus) a vector of cyclic frequency $\omega_{1}$ is directed along direction of a graviton propagation and (minus) against direction of a graviton propagation.

In spite of the fact that we have entered the graviton energy in energy-impulse tensor it does not mean that tensor began to have quantum character. Further it is necessary to take into account ideas of the quantum mechanics matrix form and to add at least to tensor components with graviton energy a factor $\exp \left(\frac{i}{\hbar} S\right)=\exp i\left(r X_{1}-\omega_{1} t\right)$ [19] where $S=\hbar \phi$ is a quantum of gravitational eikonal (70), $\phi=r X_{1}-\omega_{1} t$-its phase. We assume function of a gravitational eikonal quantum $S\left(X_{1}, t\right)$ approximately linear in a weak gravitational field.

In the matrix form of quantum mechanics $\omega_{1}$ carries the name of spectral frequency and characterizes transition of system from one quantum state in another. The factor $\exp \left(\frac{i}{\hbar} S\right)$ characterizes transition of a gravitational field quantum (graviton) on quantum states in a space-time. We shall note that at record of matrixes in the matrix form of quantum mechanics the exponential factors at a component of matrix frequently omit [19]. Thus the energy-impulse tensor will receive a kind:

$$
\begin{aligned}
T_{i k}= & \left(\begin{array}{cccc}
\rho c^{2} & T_{01} & T_{02} & T_{03} \\
T_{10} & 0 & \varepsilon \exp \left(\frac{i}{\hbar} S\right) & -\varepsilon \exp \left(\frac{i}{\hbar} S\right) \\
T_{20} & 0 & \varepsilon \exp \left(\frac{i}{\hbar} S\right) & -\varepsilon \exp \left(\frac{i}{\hbar} S\right) \\
T_{30} & 0 & \varepsilon \exp \left(\frac{i}{\hbar} S\right) & -\varepsilon \exp \left(\frac{i}{\hbar} S\right)
\end{array}\right) \\
& =\left(\begin{array}{cccc}
\rho c^{2} & T_{01} & T_{02} & T_{03} \\
T_{10} & 0 & \frac{2 \hbar \omega_{1}}{\mathrm{~V}} \exp i\left(r X_{1}-\omega_{1} t\right) & -\frac{2 \hbar \omega_{1}}{\mathrm{~V}} \exp i\left(r X_{1}-\omega_{1} t\right) \\
T_{20} & 0 & \frac{2 \hbar \omega_{1}}{\mathrm{~V}} \exp i\left(r X_{1}-\omega_{1} t\right) & -\frac{2 \hbar \omega_{1}}{\mathrm{~V}} \exp i\left(r X_{1}-\omega_{1} t\right) \\
T_{30} & 0 & \frac{2 \hbar \omega_{1}}{\mathrm{~V}} \exp i\left(r X_{1}-\omega_{1} t\right) & -\frac{2 \hbar \omega_{1}}{\mathrm{~V}} \exp i\left(r X_{1}-\omega_{1} t\right)
\end{array}\right)
\end{aligned}
$$


The energy-impulse tensor (83) has quantum character.

\section{The Graviton Equation}

For a finding of the graviton equation we shall substitute an energy-impulse tensor (83) in Einstein's equation as (21). The tensor energy-impulse trace (83) looks like $T=\rho c^{2}$. Hence the Equation (21)-the graviton equation will be transformed to a kind:

$$
R_{i k}=\frac{8 \pi k}{c^{4}}\left(T_{i k}-\frac{1}{2} g_{i k} \rho c^{2}\right)
$$

Let's consider the graviton equation in almost flat space at absence of a mass component in energy-impulse tensor (84) i.e. at $T=\rho c^{2}=0$. The gravitons practically do not bend space-time. Therefore it is possible to use the Formula (32):

The graviton radiation cross-section therefore all components in the Equation (32) with indexes 1 at the propagation of graviton in direction $X_{1}$ are excluded. Tensor components $h_{i k}$ remain only: $h_{23}$ and $h_{22}=-h_{33}$ [11]. We shall notice also that in the energy-impulse tensor (76) $T_{22}=-T_{33}$.

For a component $h_{23}$ the Equation (32) looks like:

$$
\frac{\partial^{2} h_{23}}{\partial X_{1}^{2}}-\frac{1}{c^{2}} \frac{\partial^{2} h_{23}}{\partial t^{2}}=-\frac{16 \pi k}{c^{4}} \frac{2 \hbar \omega}{\mathrm{V}} \exp i\left(r X_{1}-\omega t\right)
$$

For component $h_{22}$ a sign in the right part of the equation is positive. The index 1 at frequency is omitted.

Therefore designating $\chi=h_{i k} \mathrm{~V}$ where $i k=22,23,33$ we shall find:

$$
\frac{\partial^{2} \chi}{\partial X_{1}^{2}}-\frac{1}{c^{2}} \frac{\partial^{2} \chi}{\partial t^{2}}= \pm \frac{32 \pi k \hbar \omega}{c^{4}} \exp i\left(r X_{1}-\omega t\right)
$$

The factor before an exponent in the right part (86) is proportional to the double scalar curvature of space-time due to presence graviton, (65) $R=\frac{8 \pi k}{c^{4}} \frac{E}{\mathrm{~V}}=\frac{8 \pi k}{c^{4}} \frac{2 \hbar \omega}{\mathrm{V}}$. This factor is extremely small $\frac{32 \pi k \hbar}{c^{4}}=0.87 \times 10^{-74} \mathrm{~cm} \cdot \mathrm{s}$ that specifies almost flat space-time.

The Equation (86) describes a gravitational wave and graviton propagating from left to right therefore the general solution of the Equation (86) we shall search as:

$$
\chi=C_{1} f_{1}\left(r X_{1}-\delta t\right)+C_{2} f_{2}(t) \exp i r X_{1}
$$

where $C_{1}$ and $C_{2}$ there are constants, $f_{1}\left(r X_{1}-\delta t\right)$ and $f_{2}(t)$-any functions, $\delta$-gravitational wave frequency connected with a velocity of its propagation $r=\frac{\delta}{c}$. Frequency $\delta$ is not equal to own graviton frequency $\omega$. The first term (87) describes the gravitational wave the second term-graviton.

Let's substitute (87) in the Equation (86) which have been written down as: 


$$
\frac{\partial^{2} \chi}{\partial X_{1}^{2}}-\frac{1}{c^{2}} \frac{\partial^{2} \chi}{\partial t^{2}}= \pm \gamma \omega \exp i\left(r X_{1}-\omega t\right)
$$

where it is designated $\gamma=\frac{32 \pi k \hbar}{c^{4}}-$ a constant.

The first term (87) satisfies to the Equation (88) without the right part. Therefore after substitution we shall find:

$$
\frac{\mathrm{d}^{2} f_{2}}{\mathrm{~d} t^{2}}+\delta^{2} f_{2} \pm \frac{\gamma \omega c^{2}}{C_{2}} \exp (-i \omega t)=0
$$

The particular solution of the Equation (89) dependent on own graviton frequency $\omega$ we shall search as:

$$
f_{2}=A \exp (-i \omega t)
$$

Substituting (90) in (89) we shall find:

$$
A= \pm \frac{\gamma \omega c^{2}}{C_{2}\left(\omega^{2}-\delta^{2}\right)}
$$

Hence according to (87) the solution of the Equation (88) looks like:

$$
\chi=C_{1} f\left(r X_{1}-\delta t\right) \pm \frac{\gamma \omega c^{2}}{\left(\omega^{2}-\delta^{2}\right)} \exp i\left(r X_{1}-\omega t\right)
$$

Taking into account a designation $\chi=h_{i k} \mathrm{~V}$ where $i k=22,23,33$, and also $\gamma=\frac{32 \pi k \hbar}{c^{4}}$, and (55), (65) we shall find:

$$
\begin{aligned}
h_{i k} & =a e_{i k} \cos \left(r X_{1}-\delta t\right) \mp \frac{32 \pi k \hbar \omega e_{i k}}{c^{2}\left(\delta^{2}-\omega^{2}\right) \mathrm{V}} \exp i\left(r X_{1}-\omega t\right) \\
& =a e_{i k} \cos \left(r X_{1}-\delta t\right) \mp \frac{2 R c^{2} e_{i k}}{\left(\delta^{2}-\omega^{2}\right)} \exp i\left(r X_{1}-\omega t\right)
\end{aligned}
$$

where $e_{i k}$ there is a unit polarization tensor (56).

The top sign in (92) and (93) corresponds gravitational additive to the metric tensor $h_{22}$, and the bottom sign to $h_{23}$ and $h_{33}$; the first term is responsible for propagation of the gravitational wave the second term characterizes graviton. The size $h_{i k}$ naturally does not depend on normalizing volume V.

\section{Registration of Graviton}

The kind of function (93) allows draw some conclusions concerning interrelation of a gravitational wave and graviton. Far from massive bodies a graviton as the quantum effect is practically imperceptible. It practically has not influence on registration of the gravitational waves.

However function (93) has interesting feature. At $\omega \rightarrow \delta$ i.e. at aspiration of a graviton frequency to the gravitational wave frequency additional components of the metric tensor $h_{i k}$ aspire to infinity. The resonance of the graviton and gravitational wave frequencies is observed. This phenomenon can be used for registration of gravitons. 
Let's assume that two massive cosmic bodies (for example a double star, Figure 1) rotate around of the common mass centre. This system radiates gravitational waves $h_{i k}=a e_{i k} \cos \left(r X_{1}-\delta t\right)$ with constant frequency $\delta$ (55). If graviton gets in area of such cosmic bodies its own frequency $\omega$ grows according to the formula similar (9) for photon. According to the Formula (93) growth of a graviton energy occurs much faster $2 \hbar \omega$ that specifies resonant pumping of energy of these massive bodies gravitational field (through gravitational radiation $P$, Figure 1$)$ in a graviton. Graviton energy can considerably exceed energy of a gravitational wave and finally graviton can be registered. Probably for this purpose it is necessary to install the detector on massive bodies or near to them (on their planet or the artificial satellite). With the help of such detector it is possible to register abnormal splash in gravitation at graviton passage.

At distance from the bodies a graviton return the energy to a gravitational field of the bodies; its frequency falls (as red displacement in gravitation), Figure 1. Therefore energy of a gravitational field of the massive bodies as a whole does not change at flight near them of gravitons. We shall notice also that Riemann's curvature of space-time is positive therefore in the considered effect the additional components of matrix tensor $h_{23}$ and $h_{33}$ take part only. They resonantly grow at flight of a graviton near the massive bodies.

\section{Conclusions}

On the basis of Einstein's equation for gravitation the gravitational radiation of a double star is investigated. By refusal from a stresses tensor into energy-impulse tensor, and replacement corresponding a component in power sizes also introductions of a gravitational eikonal, the quantum quantization of gravitational radiation is lead.

The solution of the quantum equation of Einstein in the certain direction shows that this solution represents the sum of two composed, first of which characterizes gravitational waves, and the second-graviton.

At approach of the gravitons to a double star there is a resonant pumping of the gravitational field energy through gravitational radiation to gravitons. It allows registering the gravitons. At distance of the gravitons from a double star their energy comes back in a gravitational field of the stars. Frequency of the gravitons decreases as red displacement for gravitation. Therefore as a whole the gravitons do not influence on a gravitational field of stars.

\section{Conflicts of Interest}

The authors declare no conflicts of interest regarding the publication of this paper.

\section{References}

[1] Bronstein, M.P. (1936) Quantization of Gravitational Waves. Moscow. Journal of Experimental and Theoretical Physics, No. 6, 195-236.

[2] Wheeler, J.A. (1955) Geons. Physical Review, 97, 511-536. 
https://doi.org/10.1103/PhysRev.97.511

[3] Kiefer, C. (2004) Quantum Gravity. Oxford University Press, New York, 308.

[4] Rubakov, V.A. and Tinyakov, P.G. (2008) Infrared-Modified Gravities and Massive Gravitons. Uspekhi Fizicheskikh Nauk, 178, 785-822.

[5] Corda, C. (2009) Interferometric Detection of Gravitational Waves: The Definitive Test for General Relativity. International Journal of Modern Physics D, 18, 2275-2282. https://doi.org/10.1142/S0218271809015904

[6] Landau, L.D. and Lifshits, E.M. (1967) Theory of Field. Science, Moscow, 460.

[7] Kaluza T. (1979) To Problem of Physics Unity, the Collection: Albert Einstein and the Theory of Gravitation. Science, Moscow, 529-534.

[8] Pardo, K., Fishbach, M., Holz, D.E. and Spergel, D.N. (2018) Limits on the Number Spacetime Dimensions from GW170817. arXiv:1801.08160v1.

[9] Faddeev, L.D. (1998) Mathematical Physics. The Encyclopedia. In: Big Russian encyclopedia, Under Edit, Scientific Publishing House, Moscow, 153.

[10] Levich, V.G. (1962) Theoretic Physics Course. V. 1. FIZMATGIS, Moscow, 696.

[11] Zeldovich, J.B. and Novikov, I.D. (1971) The Theory of Gravitation and Evolution of Stars. Science, Moscow, 484.

[12] Peters, P.C. and Mathews, J. (1963) Gravitational Radiation from Point Masses in a Keplerian Orbit. Physical Review, 131, 435-440. https://doi.org/10.1103/PhysRev.131.435

[13] Konopleva, N.P. and Popov V.N. (2000) Gauge Fields. Editorial URSS, Moscow, 272.

[14] Fradkin, E.S. (1965) The Method of Green's Function in the Theory of the Quantum Fields and Quantum Statistics, Moscow, Works FIAN. Science, 29, 7-138.

[15] Levich, V.G., Vdovin, J.A. and Mjamlin, V.A. (1962) Theoretic Physics Course. V. 2. FIZMATGIS, Moscow, 820.

[16] Bakelman, I.J. (1967) High Geometry. Education, Moscow, 349.

[17] Volobuev, A.N. (2012) Basis of Nonsymmetrical Hydromechanics. Nova Science Publishers, Inc., New York, 198.

[18] Schlichting, H. (1974) The Boundary Layer Theory. Science, Moscow, 63.

[19] Mott, N. and Sneddon, I. (2007) Wave Mechanics and Its Applications. ComKniga, Moscow, 389. 